\title{
Eficacia de los tratamientos cognitivo-conductuales en el trastorno obsesivo-compulsivo en niños y adolescentes: una revisión cualitativa
}

\author{
Ana Isabel Rosa-Alcázar*, Marina Iniesta-Sepúlveda y Ángel Rosa-Alcázar
}

Universidad Murcia (España)

\begin{abstract}
Resumen: Se presentan los resultados de un estudio cualitativo de revisión de la eficacia de los tratamientos cognitivo-conductuales utilizados en el trastorno obsesivo-compulsivo en niños y adolescentes. Se localizaron y recuperaron un total de 84 estudios, de los cuales 17 consistían en informes de caso, 37 en diseños de caso único, 19 estudios pre-experimentales con diseño pre-postest, 6 estudios cuasi-experimentales y 5 estudios experimentales con grupo de control. El procedimiento más utilizado en los diferentes estudios ha sido la exposición con prevención de respuesta, siendo además el de mayor eficacia. Se constata la baja representación de estudios de comparación tanto cuasi-experimentales como experimentales, al igual que la escasez de medidas de seguimiento a medio y largo plazo, la falta de utilización de medidas observacionales y el escaso control de la sintomatología encubierta y trastornos comórbidos.

Palabras clave: adolescentes; trastorno obsesivo-compulsivo; tratamiento cognitivo-conductual; niños; adolescentes.
\end{abstract}

\section{Introducción}

El Trastorno Obsesivo Compulsivo (TOC, en adelante) se caracteriza por la presencia de obsesiones, que causan ansiedad y malestar significativo en el sujeto, y por compulsiones que son realizadas por el mismo con el objetivo de reducir o neutralizar la ansiedad (American Psychiatric Association, 2000). El DSM-IV-TR informa que los estudios en la población general realizados en niños/as y adolescentes han estimado una prevalencia global del $1 \%$ al $2.3 \%$ y una prevalencia anual del $0.7 \%$. En España, la incidencia del TOC fue estudiada por Canals, Doménech, Carbajo y Blade (1997) en una muestra de jóvenes de 18 años hallando un $0.7 \%$ en base a los criterios del DSM-III-R y un 1.4\% siguiendo el enfoque CIE-10. Datos recientes sugieren que en muestras no clínicas cerca de un 10\% de niño/as con 11 años tienen síntomas obsesivo-compulsivos (Fullana et al., 2009).

Las consecuencias de este trastorno en la vida de los niños/adolescentes son enormemente graves, interfiriendo en su vida diaria (en los juegos, en el rendimiento escolar, en la relación con los amigos, en casa, etc.), llegando a dominar la vida de un sujeto, perdiendo la mayoría de su tiempo en los rituales. Las complicaciones a corto, medio y largo plazo son variadas; en concreto, presentan dificultades escolares en el aprendizaje o en la relación social, trastornos del humor, síndrome de agotamiento seguido de mayor obstinación en los rituales, incidiendo en el sueño y en la alimentación, presentando secuelas físicas derivadas de los rituales tales como dermatitis, y pudiendo llegar a tentativas de suicidio (Toro, 2006)

* Dirección para correspondencia [Correspondence address]: Ana Isabel Rosa Alcázar. Departamento de Personalidad, Evaluación y Tratamiento Psicológicos. Facultad de Psicología, Campus Universitario de Espinardo, Universidad de Murcia, Apdo. 4021, 30080 Espinardo, Murcia (España).E-mail: airosa@um.es

\begin{abstract}
Title: Effectiveness of cognitive-behavioural therapy for obsessivecompulsive disorder in children and adolescents: a qualitative review.

Abstract: This paper presents the results a qualitative review study on effectiveness of cognitive-behavioural therapy for obsessive-compulsive disorder in children and adolescents. A total of 84 studies were located and retrieved, of which 17 were case reports, 37 were single case designs, 19 were quasi-experimental one-group studies, 6 were quasi-experimental comparison studies and 5 randomized controlled trials. The most widely treatment procedure, in the studies, was exposure with response prevention, which also was the most effective. We found a low representation of comparison studies, both quasi-experimental and experimental designs, as well as a lack of medium and long-term follow-ups, observational measures and a poor control of covert symptomatology and comorbidity.

Key-words: obsessive-compulsive disorder; children; adolescents; cognitive-behavioural therapy.
\end{abstract}

En los últimos años, este problema presentado en población infanto-juvenil ha recibido una mayor atención por parte de clínicos e investigadores. Esto ha conllevado una mejora de los instrumentos de evaluación y un aumento de las intervenciones terapéuticas tanto a nivel psicológico como farmacológico (Rosa-Alcázar, Olivares e Iniesta, 2010). Aunque a principios de 1970 comienzan a surgir los primeros informes, proliferando a partir de la década siguiente los estudios de caso y los diseños pretest-postest de un solo grupo, no es hasta fechas más recientes cuando encontramos diseños que están aportando el apoyo empírico necesario a las intervenciones psicológicas. Los procedimientos derivados del enfoque cognitivo-conductual han demostrado su eficacia y efectividad mediante el empleo de una metodología adecuada que permite su replicabilidad y difusión. Mientras, desde otros enfoques no se han desarrollado, hasta el momento, procedimientos terapéuticos estandarizados que hayan demostrado su eficacia en estudios empíricos.

Uno de los componentes principales del modelo cognitivo-conductual es la exposición con prevención de respuesta, procedimiento que, tal y como se ha comprobado recientemente, proporciona beneficios similares utilizado bien solo o en combinación con otras técnicas (Rosa-Alcázar, SánchezMeca, Gómez-Conesa y Marín-Martínez, 2008). Sin embargo, en muchas ocasiones, las intervenciones llevadas a cabo en el TOC infantil han incluido otros componentes además de la exposición con prevención de respuesta, como son psicoeducación neurobiológica (March y Mulle, 1998), entrenamiento a los padres o intervenciones basadas en la familia (Barrett, Farrell y March, 2004; Freeman et al., 2008), intervenciones narrativas (March y Mulle, 1998; Wagner, 2003) o técnicas cognitivas y de manejo de ansiedad (Barrett et al., 2004; March y Mulle, 1998; Pediatric OCD Treatment Study [POTS] Team, 2004). 
En este momento, nos encontramos con un número suficiente de trabajos en relación con el tratamiento del TOC, en el contexto infanto-juvenil, como para hacer posible y necesaria una revisión específica de los mismos con el fin de analizar la eficacia del tratamiento psicológico en este problema, deteniéndonos en las técnicas, instrumentos de medida y las variables más influyentes en la eficacia y eficiencia de sus efectos. Esta revisión cualitativa comprende estudios de caso, diseños de caso único, estudios de un solo grupo (pre-experimentales), y diseños de comparación entre grupos (cuasi-experimentales y experimentales). La revisión de la eficacia se justifica porque: (1) No se disponen de estudios de revisión cualitativa recientes sobre este problema que recoja con detalle los diferentes estudios empíricos en población infantil y adolescente. (2) Se precisa incrementar nuestro conocimiento respecto de los protocolos de eficacia probada existentes para esta población. (3) Por otro lado, se hace conveniente en este punto abordar la cuestión del pronóstico del TOC en estas edades, así como qué factores favorecen o dificultan la respuesta al tratamiento.

\section{Método}

\section{Materiales y procedimiento}

Los criterios conceptuales y metodológicos que nos permitieron incluir con rigor un informe de investigación fueron los siguientes: (a) Referirse al tratamiento de niños y adolescentes con TOC utilizando procedimientos diagnósticos reconocidos internacionalmente (DSM y/o CIE, en cualquiera de sus versiones), (b) aplicar algún tratamiento psicológico, (c) estar escrito en inglés, castellano o francés y (d) estar publicado entre 1970-2010.

Como procedimientos de búsqueda de los estudios se utilizaron fuentes formales e informales. En primer lugar, se realizaron búsquedas computerizadas en las bases PsycLIT (PsycINFO), CSIC-ISOC, Medline, ERIC, PsycARTICLES, Google Académico, PSICODOC y Biblioteca Cochrane Plus, comprendiendo los años 1960-2010, con las palabra-clave en inglés: Obsessive compulsive, OCD, treatment, cognitive behavioral therapy, CBT, exposure response prevention, ERP, trial, pediatric, child $^{*} y$ adolesc ${ }^{*}$; y en castellano (obsesivo-compulsivo, TOC, tratamiento, terapia cognitivo-conductual, TCC, pediátrico, exposición con prevención de respuesta, infan*, niñ* y adolesce*) buscadas en el título y en el abstract. En segundo lugar, se escribieron cartas a investigadores de reconocido prestigio solicitándoles el envío de trabajos, publicados o no. En tercer lugar, se revisaron las referencias de los metaanálisis publicados sobre el tema (Abramowitz, Whiteside y Deacon, 2005; Geller et al., 2003, O`Kearney, 2007; Watson y Rees, 2008). Finalmente, se revisaron las referencias de los estudios recuperados.

El proceso de búsqueda supuso la consulta de más de 1254 referencias, que nos permitió identificar y recuperar un total de 84 estudios, de los cuales 17 consistían en informes de caso, 37 en diseños de caso único, 19 estudios pre- experimentales con diseño pre-postest, 6 estudios cuasiexperimentales y 5 estudios experimentales con grupo de control. Los artículos seleccionados estaban escritos en inglés y castellano.

\section{Análisis de la información}

Los estudios pueden ser categorizados en cinco grupos: (1) Informes de caso, (2) Diseños de caso único $(\mathrm{N}=1)$, (3) Diseños pretest-postest sin grupo de comparación, (4) Diseños cuasi-experimentales, y (5) Diseños experimentales. A pesar de que este estudio se centra en los diseños de grupo, mencionamos algunas características generales de los informes de caso y diseños de caso único.

\section{Informes de caso y diseños de caso único}

Los informes de caso fueron realizados principalmente entre el año 1967 y finales de 1990. Se observa la heterogeneidad en la aplicación de técnicas terapéuticas encontrando la utilización de extinción, saciación, detención del pensamiento, modelado, inundación, exposición con prevención de respuesta (EPR, en adelante), reforzamiento diferencial de conductas, técnicas cognitivas y terapia familiar. La edad de los sujetos que recibieron los tratamientos descritos en estos informes ha variado entre los 6 y los 16 años. Respecto de la eficacia, todos han informado de resultados positivos en cuanto a la respuesta a la intervención, sin embargo, ésta no ha sido medida de forma cuantitativa.

En cuanto a los diseños de caso único, también presentes desde principios de la década de 1970 hasta la actualidad, presentan notables mejoras metodológicas respecto a los comentados con anterioridad. Los diseños utilizados para evaluar la eficacia han sido del tipo: A-B, A-B-A-B, línea base múltiple y serie de casos. Las intervenciones han consistido en técnicas operantes, extinción, EPR, inundación, modelado, terapia cognitiva, entrenamiento a padres en manejo de contingencias y biblioterapia. A partir de 1990, se comienzan a evaluar mediante este tipo de diseños, distintos paquetes de tratamiento multicomponente, los cuales se componen en general de EPR y técnicas cognitivas, con o sin implicación de la familia. Asimismo encontramos tanto la modalidad de aplicación semanal como la intensiva. Muchos de los programas de tratamiento aplicados se basan en protocolos o manuales estandarizados diseñados específicamente para estas edades como son los de March y Mulle (1998), Lewin, Storch, Merlo, Murphy y Geffken (2005), Wagner (2003) y Piacentini, Jacobs, Maidment y Bergman (1998). En cuanto a la administración de otros tratamientos, son varios los estudios que informan de la toma concurrente de psicofármacos, en concreto, algún tipo de ISRS, clomipramina, risperidona, clonacepan y quietiapina. El rango de edad de los sujetos es más amplio que el citado con anterioridad, oscilando entre 5 y 18 años. Con algunas excepciones (Clark, 1982; Piacentini, Gitow, Jaffer, Graae y Whitaker, 1994) se informa de la mejora de todos los sujetos tras la aplicación 
de los tratamientos, midiendo la mejora en porcentajes de reducción de la sintomatología entre el $42.5 \%$ y $100 \%$. La información referente a la eficacia durante periodos de seguimiento es muy escasa siendo tan solo dos los informes que presentan estos datos mostrando resultados favorables en un caso (Whiteside, Brown y Abramowitz, 2008) y negativos en otro (Whiteside y Abramowitz, 2006). Las diferentes medidas de mejora utilizadas según el estudio, han consistido en instrumentos clínicos como la Escala Yale-Brown de Obsesiones y Compulsiones para Niños (CY-BOCS; Scahill et al., 1997), la Entrevista Diagnóstica del Instituto Nacional de Salud Mental para Niños (NIMH; Shaffer, Fisher, Lucas, Dulcan y Schwab-Stone, 2000) o la Escala de Impresión Clínica Global-Severidad/Mejora (CGI-S/I; Guy, 1978; National Institute of Mental Health, 1985). Además de otros autoinformes, como la Escala de Impacto del Trastorno Obsesivo-Compulsivo para Niños (COIS; Piacentini y Jaffer, 1999) y medidas de registro observacionales de frecuencia de los síntomas o de nivel de ansiedad experimentado.

\section{Diseños pretest-postest sin grupo de comparación}

La evidencia empírica informada por los numerosos estudios pre-postest acerca de la eficacia del tratamiento cognitivo conductual del TOC en la infancia y la adolescencia es muy amplia. Desde principios de 1980, diferentes aspectos del tratamiento han sido abordados en estos estudios, entre ellos encontramos: el tipo de técnica utilizada, la eliminación o ampliación de componentes a la intervención, la implicación familiar en el tratamiento y la modalidad de tratamiento, ya sea individual/grupal o intensiva/semanal. También la influencia de otros factores en la respuesta al tratamiento, como la temática de las obsesiones, la presencia de infecciones por estreptococos o la comorbilidad. A mediados de la década de 1990, se observa una mejora en la metodología de estudio referente a la introducción de instrumentos de evaluación validados y específicos para la población infantil y adolescente cuyo avance más importante es el surgimiento de la Escala de Yale-Brown de Obsesiones y Compulsiones para niños (Children's Yale-Brown Obsessive-Compulsive Scale, CY-BOCS; Scahill et al., 1997), el aumento de la homogeneidad de los tratamientos con la utilización de manuales estandarizados o la realización de los ensayos en muestras con mayor número de sujetos.

Como se ha indicado, los primeros trabajos realizados para evaluar los efectos del tratamiento cognitivo-conductual del TOC infantil con diseños pre-postest se caracterizan por la utilización de intervenciones terapéuticas individualizadas y heterogéneas, su aplicación en muestras reducidas y el uso de criterios de mejora cualitativos. Es por ello que estos primeros trabajos aportan evidencia empírica acerca de la conveniencia de intervenir sobre el TOC a edades tempranas poniendo el énfasis en las técnicas de tratamiento cognitivoconductuales.

En este marco encontramos el trabajo de Bolton, Collings y Steinberg (1983) que informa de 15 casos de TOC adolescentes tratados en régimen ambulatorio o mediante ingreso en los hospitales de Bethlem Royal y Maudsley en Reino Unido. En los casos de tratamiento ambulatorio en los que los niños presentaban alta motivación, la técnica elegida fue la práctica de la auto-exposición con prevención de respuesta. En los casos que no cumplían este requisito, se aplicó EPR con participación de los padres bien de forma pasiva (eliminando su implicación en los rituales), bien de forma activa (restringiendo las compulsiones del adolescente de forma verbal o física). Los resultados informaron de la mejora de 13 de los 15 pacientes tras la aplicación del tratamiento, mostrándose 7 de los casos libres de síntomas y 6 leves. En 1995, Bolton, Luckie y Steinberg publican el seguimiento de 14 de estos pacientes, realizado entre 9 y 14 años después encontrando que 6 de los casos mantenían el diagnostico de TOC según el DSM-III-R. El porcentaje de recuperación fue del 57\%. En esta misma línea, Apter, Bemhout y Tyrano (1984) publican un informe acerca del tratamiento de 8 jóvenes que padecían un TOC severo. Los medios de tratamiento utilizados fueron EPR, terapia familiar y medicación.

Desde mediados de 1990, los estudios comienzan a incorporar importantes mejoras metodológicas permitiendo la estandarización de las intervenciones y una mayor exactitud en la cuantificación de los resultados, aunque se continúan utilizando tamaños muestrales reducidos. Los objetivos son principalmente evaluar la eficacia de la terapia cognitivoconductual (TCC, en adelante) controlando los efectos de la medicación (ej., estabilizando las dosis) o simplificando los programas de tratamiento para evaluar la eficacia diferencial entre diferentes componentes de dicha terapia. Uno de los primeros trabajos realizados para poner a prueba un programa de tratamiento cognitivo conductual estructurado y homogéneo fue el de March, Mulle y Herbel (1994). En este estudio, los autores realizaron una evaluación preliminar de su protocolo que incluyó varios componentes: Psicoeducación, trazado de un mapa del TOC, EPR, entrenamiento en el manejo de la ansiedad (relajación, técnicas de respiración, auto-instrucciones y estrategias de afrontamiento) y sesiones familiares. El protocolo fue aplicado a una muestra de 15 niños y adolescentes entre 8 y 18 años. Los resultados mostraron que el $80 \%$ de los pacientes respondieron al tratamiento. Asimismo se pudieron observar beneficios similares a los 7 meses de seguimiento. Posteriormente, Scahill, Vitulano, Brenner, Lynch y King (1996), aplicaron la TCC a un grupo de 7 niños y adolescentes entre 10 y 15 años $(M=13)$. Los resultados mostraron una mejoría media del 61\% (rango 30$90 \%$ ). Estos cambios resultaron significativos y fueron mantenidos durante el seguimiento a corto plazo al mes y a los tres meses (media de mejoría 51\%). Dos años más tarde, Fischer, Himle y Hanna (1998) informan de la aplicación exitosa de la TCC en formato grupal. Los participantes en el estudio fueron 15 adolescentes entre 12 y 17 años (M =14.5). Todos ellos recibieron un programa de TCC grupal de 7 sesiones semanales, de una hora y media. El programa incluyó psicoeducación, técnicas cognitivas para la externa- 
lización del TOC, autoinstrucciones y EPR autoaplicada o guiada por el terapeuta. Los sujetos mejoraron significativamente del pretest al postest y de éste al seguimiento. En 1998, March y Mulle publican el primer manual estandarizado de tratamiento cognitivo-conductual para el TOC en la infancia y la adolescencia. Una primera adaptación de este protocolo, adicionando el componente familiar, fue puesta a prueba por Waters, Barret y March (2001) en un ensayo abierto en el que participaron 7 niños entre 10 y 14 años. El programa de TCC en este estudio, denominado POWER (Positively Overcoming Worries and Excessive Rituals), incluyó 14 sesiones de 90 minutos de duración de los cuales, en general, 45 fueron dedicados a la terapia con el niño, 30 al entrenamiento a padres y 15 para la revisión y asignación de las tareas para casa. Durante las sesiones se utilizó un libro de trabajo para niños y padres. El trabajo realizado con el niño consistió en psicoeducación, entrenamiento cognitivo y manejo de ansiedad y EPR. El componente familiar del programa incluyó: Formación de un equipo de apoyo, entrenamiento en reforzamiento diferencial de conducta, participación en entrenamiento en relajación del niño, reducción de la acomodación familiar, manejo de la ansiedad de los padres, participación en los ejercicios de exposición del niño y entrenamiento en resolución de problemas. Los resultados en el postest, mostraron diferencias significativas con respecto al pretest en todas las medidas. Además, en el postest, 6 de los niños no cumplían los criterios para el TOC con una reducción media del $60 \%$ en la severidad del desorden según la CY-BOCS y la NIMH. Estas ganancias fueron mantenidas a los 3 meses de seguimiento. Thienemann, Martin, Cregger, Thompson y Dyer-Friedman (2001) también evaluaron la eficacia del protocolo realizado por March y Mulle (1998) adaptando éste al formato grupal, en una muestra de 18 adolescentes con TOC entre 13 y 18 años (Media = 15.2). Los resultados informaron de mejoras significativas en la sintomatología del TOC. Piacentini, Bergman, Jacobs, Mccraken y Kretchman (2002) aumentaron la evidencia acerca de la eficacia de su protocolo mediante la realización de un estudio con una amplia muestra conformada por 42 niños y adolescentes con TOC entre 5 y 17 años $(M=11.8)$. Un 52\% de la muestra también se encontraba tomando medicación para el TOC, aunque no se observaron diferencias significativas en respuesta al tratamiento con los que no lo hacían. Tras el tratamiento, el 79\% de los sujetos respondió al mismo, observando una mejora en el CGI-I. Además en el NIMHGlobal se produjo una reducción media del 45\%.

En los estudios comentados hasta el momento, la mayoría de los participantes habían estado o estaban recibiendo algún tipo de tratamiento para el TOC, especialmente de tipo farmacológico, el cual se solía mantener durante el periodo del estudio, lo que hacía imposible la atribución de los cambios exclusivamente a las TCC. Ante esto, Benazon, Ager y Rosenberg (2002) evaluaron la eficacia de la TCC en 16 sujetos entre 8 y 17 años, que no habían recibido tratamiento para el TOC previamente. El tratamiento utilizado por estos autores se basaba en la integración de los manuales de March y Mulle (1998) y de Schwartz, (1996) e incluía 12 sesiones de una hora. Los resultados obtenidos avalaron la eficacia de la TCC sin el uso de medicación concomitante.

En el Reino Unido, Williams, Salkovskis, Forrester y Allsopp (2002) evaluaron la eficacia de la terapia cognitiva mediante su aplicación a 6 adolescentes entre 12 y 17 años. El programa de tratamiento incluyó psicoeducación, reestructuración cognitiva y experimentos conductuales. Los resultados informaron de mejoras significativas. Himle, Fischer, Van Etten, Janeck y Hanna (2003) examinaron la influencia de la presencia de tics en la respuesta a la TCC. Para ello aplicaron un programa cognitivo-conductual durante siete semanas a un grupo niños y adolescentes con TOC y tics asociados $(\mathrm{n}=8)$ y a otro grupo con TOC sin tics comórbidos $(\mathrm{n}=11)$ con edades comprendidas entre los 12 y 17 años. El programa de TCC administrado incluyó psicoeducación, EPR, estrategias cognitivas e implicación familiar. Tras el tratamiento se observaron reducciones significativas de las puntuaciones de la CY-BOCS en ambos grupos, además no se observaron diferencias significativas entre ellos en cuanto a la respuesta a la TCC.

Martin y Thienemann (2005) volvieron a poner a prueba el tratamiento grupal basado en el protocolo de March y Mulle (1998) en 14 niños de 8 a 14 años $(M=11.3)$, aumentando el grado de implicación parental. El contenido y las técnicas de tratamiento llevadas a cabo no difirieron de lo presentado en su anterior estudio (Thienemann et al., 2001). No obstante, hubo diferencias en la estructura del programa ya que los padres y sus hijos eran atendidos simultáneamente por diferentes terapeutas durante la primera hora de la sesión. Además, durante los 30 minutos restantes se trabajaba conjuntamente con ambos grupos. En cuanto a los resultados, las puntuaciones informadas por niños y padres se vieron reducidas significativamente en una media de $24.76 \%$, experimentándose en seis de los pacientes una reducción mayor al $25 \%$.

Como comentamos previamente, uno de los aspectos estudiados en este área es la influencia que ciertas variables pueden ejercer sobre la respuesta del sujeto al tratamiento cognitivo-conductual. Uno de estos factores es el subtipo de TOC que padece el sujeto, acerca del cual se han hecho diferentes clasificaciones (según la temática, la presencia o no de compulsiones y obsesiones, que se trate o no de PANDAS, etc.). Storch et al. (2006) evaluaron la eficacia de la TCC en sujetos que presentaban un TOC del tipo PANDAS (Desordenes Neuropsiquiátricos Pediátricos Asociados con Estreptococos). Se aplicó la TCC a 7 sujetos con TOC de entre 9 y 13 años. El tratamiento fue de tipo intensivo e incluyó 14 sesiones de 90 minutos a lo largo de tres semanas, basado en el manual de Lewin et al. (2005), adaptación del protocolo de March y Mulle (1998), para ser aplicado en formato intensivo. Las técnicas utilizadas fueron psicoeducación, entrenamiento cognitivo y EPR. Los padres asistieron a las sesiones y se trató la influencia de las interacciones familiares desadaptativas como la acomodación. Todos los sujetos completaron el tratamiento, mostrando una reduc- 
ción significativa $(68 \%)$ de la puntuación media en la CYBOCS, la cual se mantuvo en gran parte a los 3 meses de seguimiento (46\%). Este estudio aporta evidencia preliminar de la eficacia de la TCC en el tratamiento del TOC infantil subtipo PANDAS pudiendo ser una alternativa a otros procedimientos más invasivos utilizados para tratar este subtipo, como la inmunoglobulina intravenosa y el intercambio plasmático (Perlmutter, Leitman, Garvey, Hamburger, Feldman y Leonard, 1999).

Valderhaug, Larssona, Götestamb y Piacentini (2007) quisieron evaluar una vez más la eficacia de la TCC utilizando un diseño de un sólo grupo en contexto de la práctica clínica no académica y si la aplicación de tratamientos estandarizados y manualizados era viable en estas clínicas comunitarias. Para ello, se incluyeron en el estudio 24 niños y adolescentes entre 8-17 años $(\mathrm{M}=13.3)$ con diagnóstico primario de TOC según DSM-IV que acudieron a tres clínicas del oeste y centro de Noruega. El 92\% no estaba tomando medicación. Para el tratamiento se utilizó el manual desarrollado por Piacentini et al. (1998), el cual consistió en 12 sesiones semanales a excepción de las dos últimas que se administraron de forma quincenal. Los padres contaban con la posibilidad de participar en todas las sesiones, en las dos primeras eran recibidos junto al niño y en las restantes se dedicaban 45 minutos a la atención del niño en solitario y entre 15 y 30 minutos a los padres. El tratamiento consistió esencialmente en la aplicación de la EPR, aunque también se utilizó la psicoeducación y la aplicación de recompensas para motivar y ayudar al niño a afrontar su ansiedad durante la EPR. Tras la finalización del tratamiento, se obtuvieron mejoras significativas en los niños.

Storch et al. (2008) analizaron la respuesta diferencial al TCC de los diferentes subtipos de TOC en niños y adolescentes, Para ello, reunieron una muestra de 92 niños y adolescentes entre 7 y 19 años $(M=13.6)$ a los que se aplicó la TCC, tras contabilizar el porcentaje de sujetos que presentaban cada una de las siguientes dimensiones sintomáticas: orden/simetría, contaminación/limpieza, obsesiones sexuales/religiosas, agresividad/comprobación y acumulación. Aproximadamente, el $50 \%$ de la muestra eran participantes de otro estudio (Storch et al., 2007). Cuarenta y ocho de los sujetos recibieron tratamiento en formato semanal y 42 lo hicieron de forma intensiva. Según la GCI-I, el 76\% de los participantes respondieron al tratamiento. En cuanto la relación de la respuesta al tratamiento con las diferentes dimensiones de síntomas, se observó que los sujetos que presentan agresividad/comprobación tenían mayor probabilidad de responder al tratamiento que aquéllos que no presentaban síntomas en esta dimensión. No se encontraron diferencias significativas en aquellos sujetos que padecían síntomas en cualquier otra dimensión de las citadas. En este mismo año, Bjorgvinsson et al. (2008) trataron a 23 adolescentes de entre 13 y 17 años, en régimen hospitalario. El programa terapéutico incluyó 90 minutos de EPR asistida por el terapeuta por las mañanas, mientras que por la tarde los sujetos se autoexponían durante 60 minutos, tres veces por semana. Tam- bién se incluyo psicoeducación, técnicas cognitivas y terapia familiar individualizada. Los pacientes recibieron además tratamiento farmacológico con ISRS aunque los autores no especificaron ni los agentes activos ni las dosis administradas. Los resultados informaron de que un $70 \%$ de los pacientes cumplieron los criterios de mejora establecidos.

Merlo, Lehmkuhl, Geffken y Storch (2009) analizaron la disminución de la variable acomodación familiar tras la aplicación de la TCC en 54 niños y adolescentes. Tras el tratamiento se produjo un decremento significativo de la acomodación familiar.

Una vez observada ampliamente la eficacia de la terapia cognitivo-conductual por los numerosos estudios mencionados, los dos últimos trabajos realizados a este respecto han evaluado las mejoras en el acceso de los pacientes a la TCC, mediante su administración en formato telefónico o la viabilidad de su aplicación en el ámbito comunitario. Éste es el caso del estudio realizado por Turner, Heyman, Futh y Lovell (2009) en el cual, 10 participantes entre 13 y 17 años recibieron TCC telefónica semanal, basada en un manual estandarizado para niños (no se especifica) usado cotidianamente en el tratamiento cara a cara. Los resultados mostraron una disminución significativa de las puntuaciones en la CY-BOCS, manteniendo las mejoras a los 12 meses de seguimiento un $70 \%$ de los sujetos. Un último estudio realizado muy recientemente (Farrell, Schlup y Boschen, 2010), evalúa la efectividad de la TCC-Familiar presentada en el estudio de Barrett et al. (2004) mediante su aplicación a 35 niños y adolescentes entre 7 y 17 años. La intervención fue llevada a cabo en el contexto de las clínicas comunitarias no académicas, con el objetivo de comprobar la extrapolabilidad de los resultados exitosos de la TCC a este ámbito. Los resultados informaron de mejoras significativas tanto en sintomatología obsesivo-compulsiva como en ansiedad y depresión. En la Tabla 1 se presenta un resumen de algunas de las variables de los estudios descritos en este apartado.

\section{Diseños cuasi-experimentales}

El primer estudio encontrado que utiliza este tipo de diseño es realizado por Franklin et al. (1998), analizando la eficacia de la TCC, el control de los efectos de la medicación y las diferencias entre la modalidad intensiva y semanal. Para ello utilizaron una muestra de 14 niños y adolescentes entre 10 y 17 años $(M=14.1)$ con TOC, distribuidos bien a la modalidad intensiva de tratamiento ( $\mathrm{n}=7$, media de 18 sesiones en un mes), bien a la semanal ( $\mathrm{n}=7$, media de 16 sesiones en cuatro meses). El componente principal de ambas modalidades fue la EPR asistida por el terapeuta en consulta y practicada en el hogar del niño entre las sesiones. Tanto padres como niños recibieron psicoeducación aunque el grado de implicación de los padres fue diferente en cada caso según la conveniencia estimada por el clínico. Los programas también incluyeron el componente prevención de recaídas. Los resultados mostraron diferencias significativas entre pretest y postest y entre pretest y seguimiento con una 
reducción media de las puntuaciones en la CY-BOCS del $67 \%$ y el $62 \%$ en pretest y seguimiento, respectivamente. Según esta misma escala, el $86 \%$ de los pacientes mostraron respuesta al tratamiento (reducción $>50 \%$ ), encontrándose resultados similares en el seguimiento con un $83 \%$ de respondientes. Los resultados según el tipo de tratamiento en la CY-BOCS informaron de reducciones en el pos-test del $70 \%$ y $64 \%$ y del $58 \%$ y $67 \%$ en el seguimiento, para el tratamiento intensivo y semanal, respectivamente. En cuanto a la valoración de los efectos de la medicación, el grupo que recibió EPR en solitario mostró una reducción del $84 \%$ y del $68.8 \%$ en la CY-BOCS en postest y seguimiento respectivamente, frente al grupo EPR combinado con medicación en el cual se observaron reducciones del 55\% y 59\% en postest y seguimiento, respectivamente. A la vista de estos resultados los autores sugirieron que la TCC es un tratamiento útil combinado o no con medicación.

Tabla 1. Diseños pretest-postest de un solo grupo.

\begin{tabular}{|c|c|c|c|c|c|c|c|c|}
\hline Estudio & Edad & $\mathrm{N}$ & $\begin{array}{l}\text { Criterio Diagnos- } \\
\text { tico }\end{array}$ & Tratamiento & $\begin{array}{l}\text { Otros trata- } \\
\text { mientos }\end{array}$ & $\begin{array}{l}\text { Medidas } \\
\text { Dependientes }\end{array}$ & $\begin{array}{l}\text { Reducción en la } \\
\text { sintomatología (\%) }\end{array}$ & $\begin{array}{l}\text { Respuesta al trata- } \\
\text { miento }(\%)\end{array}$ \\
\hline $\begin{array}{l}\text { Bolton et al. } \\
\text { (1983) }\end{array}$ & $12-18$ & 15 & DSM-III-R & $\begin{array}{l}\text { EPR } \\
\text { Según el caso: } \\
\text { - Asistida por pa- } \\
\text { dres/terapeuta } \\
\text { - Régimen hospitala- } \\
\text { rio/ambulatorio }\end{array}$ & $\begin{array}{l}\text { Comipramina } \\
(26.7 \%) \\
\text { Psicoterapia } \\
\text { no dinámica } \\
(13.3 \%)\end{array}$ & $\begin{array}{l}\text { Tiempo ocupado } \\
\text { por los síntomas }\end{array}$ & No informa & $\begin{array}{l}86.6 \% \\
\text { (Libres de síntomas o } \\
\text { tiempo ocupado por } \\
\text { estos, menor a } 1 \mathrm{~h} . \text { ) }\end{array}$ \\
\hline $\begin{array}{l}\text { Apter et al. } \\
\text { (1984) }\end{array}$ & - & 8 & - & EPR & $\begin{array}{l}\text { Psicoterapia } \\
\text { no especifica } \\
\text { Medicación }\end{array}$ & - & $\begin{array}{l}\text { (Sin respuesta a la tera- } \\
\text { pia conductual) }\end{array}$ & $\begin{array}{l}\text { (Sin respuesta a la te- } \\
\text { rapia conductual) }\end{array}$ \\
\hline $\begin{array}{l}\text { March et al. } \\
\text { (1994) }\end{array}$ & $8-18$ & 15 & DSM-III-R & TCC-Individual & $\begin{array}{l}\text { ISR } \\
(93 \%)\end{array}$ & $\begin{array}{l}\text { Y-BOCS } \\
\text { NIMH-Global } \\
\text { CGI }\end{array}$ & 50.21 (CY-BOCS) & $\begin{array}{l}80 \text { (cambio } \geq 30 \% \text { en } \\
\text { CY-BOCS o NIMH) }\end{array}$ \\
\hline $\begin{array}{l}\text { Scahill et al. } \\
(1996)\end{array}$ & $10-15$ & 7 & DSM-III-R & $\begin{array}{l}\text { EPR con implicación } \\
\text { familiar }\end{array}$ & $\begin{array}{l}\text { Medicación } \\
(71 \%)\end{array}$ & CY-BOCS & 61 (CY-BOCS) & No informa \\
\hline $\begin{array}{l}\text { Fischer et al. } \\
\text { (1998) }\end{array}$ & $12-17$ & 15 & DSM-III-R & TCC Grupal & $\begin{array}{l}\text { Medicación } \\
(67 \%)\end{array}$ & CY-BOCS & $\begin{array}{l}\text { Postest } \\
32 \text { (CY-BOCS) } \\
\text { Seguimiento } \\
46 \text { (CY-BOCS) }\end{array}$ & No informa \\
\hline $\begin{array}{l}\text { Waters et al. } \\
\text { (2001) }\end{array}$ & $10-14$ & 7 & DSM-IV & TCC Familiar Individual & No informa & $\begin{array}{l}\text { CY-BOCS } \\
\text { NIMH-OCS }\end{array}$ & $\begin{array}{l}60 \text { (CY-BOCS, NIMH- } \\
\text { OCS) }\end{array}$ & $\begin{array}{l}86 \text { (No cumplimiento } \\
\text { de criterios diagnósti- } \\
\cos \text { ) }\end{array}$ \\
\hline $\begin{array}{l}\text { Thienemann } \\
\text { et al. (2001) }\end{array}$ & $13-18$ & 18 & DSM-IV & TCC Grupal & $\begin{array}{l}\text { Medicación } \\
(83 \%)\end{array}$ & $\begin{array}{l}\text { CY-BOCS } \\
\text { NIMH } \\
\text { CGI }\end{array}$ & 25 (CY-BOCS) & $\begin{array}{l}50 \text { (Cambio } \geq 25 \% \text { en } \\
\text { CY-BOCS) }\end{array}$ \\
\hline $\begin{array}{l}\text { Williams et } \\
\text { al. (2002) }\end{array}$ & $12-17$ & 6 & DSM-IV & Terapia cognitiva & $\begin{array}{l}\text { Medicación } \\
(16.67 \%)\end{array}$ & $\begin{array}{l}\text { CY-BOCS } \\
\text { OCI }\end{array}$ & 40 (CY-BOCS) & No informa \\
\hline $\begin{array}{l}\text { Piecentini et } \\
\text { al. }(2002)\end{array}$ & $7-17$ & 42 & DSM-IV & TCC Individual & $\begin{array}{l}\text { Medicación } \\
(52 \%)\end{array}$ & $\begin{array}{l}\text { CGI } \\
\text { NIMH } \\
\end{array}$ & 45 (NIMH-OCS) & $78.6($ CGI-S < 2) \\
\hline $\begin{array}{l}\text { Benazon et } \\
\text { al. (2002) }\end{array}$ & $8-17$ & 16 & DSM-IV & TCC Individual & Ninguno & $\begin{array}{l}\text { CY-BOCS } \\
\text { CGI } \\
\text { NIMH-OCS }\end{array}$ & 47.6 (CY-BOCS) & $\begin{array}{l}81 \text { (No informa de } \\
\text { criterio) }\end{array}$ \\
\hline $\begin{array}{l}\text { Himle et al. } \\
(2003)\end{array}$ & $12-17$ & 19 & $\begin{array}{l}\text { DSM-III-R } \\
\text { DSM-IV }\end{array}$ & TCC Individual & $\begin{array}{l}\text { Medicación } \\
(68 \%)\end{array}$ & CY-BOCS & 30.36 (CY-BOCS) & No informa \\
\hline $\begin{array}{l}\text { Martin y } \\
\text { Thienemann } \\
\text { (2005) }\end{array}$ & $8-14$ & 14 & DSM-IV & TCC Familiar Grupal & $\begin{array}{l}\text { Medicación } \\
(64 \%)\end{array}$ & $\begin{array}{l}\text { CY-BOCS } \\
\text { NIMH-OCS } \\
\text { CGI }\end{array}$ & 24.76 (CY-BOCS) & No informa \\
\hline $\begin{array}{l}\text { 'Storch et al. } \\
\text { (2006) }\end{array}$ & $9-13$ & 7 & DSM-IV & TCC Intensivo & $\begin{array}{l}\text { ISRS } \\
(86 \%)\end{array}$ & $\begin{array}{l}\text { ADIS-IV-P } \\
\text { CY-BOCS } \\
\text { CGI }\end{array}$ & $\begin{array}{l}\text { Postest } \\
68 \text { (CY-BOCS) } \\
\text { Seguimiento } \\
46 \text { (CY-BOCS) }\end{array}$ & $\begin{array}{l}\text { Postest } \\
86 \\
\text { Seguimiento } \\
50 \\
(\text { CGI-S }<2)\end{array}$ \\
\hline $\begin{array}{l}\text { Valderhaug } \\
\text { et al. (2007) }\end{array}$ & $8-17$ & 24 & DSM-IV & $\begin{array}{l}\text { TCC Familiar } \\
\text { (Clínicas comunitarias) }\end{array}$ & $\begin{array}{l}\text { Medicación } \\
(8.3 \%)\end{array}$ & $\begin{array}{l}\text { CY-BOCS } \\
\text { CGI }\end{array}$ & $\begin{array}{l}\text { Postest } \\
60.6 \text { (CY-BOCS) } \\
\text { Seguimiento } \\
68.8 \text { (CY-BOCS) }\end{array}$ & $\begin{array}{l}\text { Postest } \\
91.7 \\
\text { Seguimiento } \\
100 \\
(\text { CGI-I } \geq 6)\end{array}$ \\
\hline $\begin{array}{l}\text { Storch, } \\
\text { Merlo, Lar- } \\
\text { son, Bloss } \\
\text { et al. (2008) }\end{array}$ & $7-19$ & 92 & DSM-IV & TCC Intensivo o semanal & $\begin{array}{l}\text { Medicación } \\
(64 \%)\end{array}$ & $\begin{array}{l}\text { CY-BOCS } \\
\text { CGI }\end{array}$ & $\begin{array}{l}\text { (CY-BOCS) } \\
\text { Acumulación } \\
49.7 \\
\text { Orden/simetría } \\
56.5 \\
\text { Contaminación } \\
56.5 \\
\text { Sexo/religión }\end{array}$ & $76($ CGI-I $\leq 2)$ \\
\hline
\end{tabular}




\begin{tabular}{|c|c|c|c|c|c|c|c|c|}
\hline & & & & & & & $\begin{array}{l}57.1 \\
\text { Agresión/ comproba- } \\
\text { ción } \\
59.6\end{array}$ & \\
\hline $\begin{array}{l}\text { Bjorgvins- } \\
\text { son et al. } \\
\text { (2008) }\end{array}$ & 3-17 & 23 & No informa & $\begin{array}{l}\text { TCC } \\
\text { (Régimen hospitalario) }\end{array}$ & $\begin{array}{l}\text { Terapia Fami- } \\
\text { liar } \\
\text { Medicación }\end{array}$ & CY-BOCS & 40.2 (CY-BOCS) & $\begin{array}{l}70 \text { (mejora clínica y } \\
\text { estadísticamente sig- } \\
\text { nificativa) }\end{array}$ \\
\hline $\begin{array}{l}\text { Turner et al. } \\
(2009)\end{array}$ & $13-17$ & 10 & CIE-10 & TCC Telefónica & $\begin{array}{l}\text { Medicación } \\
(50 \%)\end{array}$ & $\begin{array}{l}\text { CY-BOCS } \\
\text { CHOCI }\end{array}$ & $\begin{array}{l}\text { Postest } \\
53 \text { (CY-BOCS) } \\
\text { Seguimiento } \\
58 \text { (CY-BOCS) }\end{array}$ & $\begin{array}{l}70 \text { (Cambio } \geq 10 \% \\
\text { en CY-BOCS) }\end{array}$ \\
\hline $\begin{array}{l}\text { Merlo et al. } \\
(2009)\end{array}$ & $6-18$ & 49 & DSM-IV & TCC Intensivo o semanal & $\begin{array}{l}\text { Medicación } \\
(76 \%)\end{array}$ & $\begin{array}{l}\text { CY-BOCS } \\
\text { FAS }\end{array}$ & $\begin{array}{l}\text { CY-BOCS } \\
63.6 \\
\text { FAS } \\
50 \\
\end{array}$ & $\begin{array}{l}80 \text { (Cambio } \geq 30 \% \text { o } \\
\text { puntuación }<16 \text { en } \\
\text { CY-BOCS) }\end{array}$ \\
\hline $\begin{array}{l}\text { Farrell et al. } \\
(2010)\end{array}$ & $7-17$ & 35 & DSM-IV & $\begin{array}{l}\text { TCC Familiar } \\
\text { (Clínicas Comunitarias) }\end{array}$ & $\begin{array}{l}\text { Medicación } \\
(17.1 \%)\end{array}$ & $\begin{array}{l}\text { NIMH-OCS } \\
\text { CY-BOCS } \\
\text { CGI }\end{array}$ & 61 (CY-BOCS) & 63 (ADIS) \\
\hline
\end{tabular}

CHOCI: Inventario Obsesivo Compulsivo para Niños; ADIS: Protocolo de Entrevista de Trastornos de Ansiedad del DSM; CY-BOCS: Escala Yale-Brown de Obsesiones y Compulsiones para Niños; LOI-CV: Inventario de Obsesiones de Leyton; CGI: Escala de Impresión Clínica Global; FAS: Escala de Acomodación Familiar para el Trastorno Obsesivo-Compulsivo; NIMH: Entrevista del National Institute of Mental Health Diagnostic for Children Version IV; TCC: Terapia cognitivo-conductual; EPR: Exposición con prevención de respuesta.

Son escasos los estudios que han comparado de forma directa la eficacia de la terapia psicológica frente a la aportada por la terapia psicofarmacología en el tratamiento de niños y adolescentes con TOC. El primer estudio que compara tratamiento psicológico y farmacológico fue el llevado a cabo por de Haan, Hoogduin, Buitelaar y Keijsers (1998), en el que 22 niños fueron asignados aleatoriamente al tratamiento con clomipramina o EPR durante 12 semanas. La EPR fue aplicada de forma gradual tras una sesión de psicoeducación. También se incluyeron técnicas cognitivas. Los resultados mostraron la eficacia de ambos tratamientos para reducir la sintomatología en todas las medidas; sin embargo, la EPR se mostró significativamente más eficaz que la clomipramina, tanto en el porcentaje de sujetos que respondieron al tratamiento $(66.7 \%$ con EPR; $50 \%$ con clomipramina) como en la reducción media de la severidad del desorden (59.9\% con EPR; $33.4 \%$ con clomipramina 33.4\%) en la CY-BOCS. En 2005, Ramos-Asbarh et al. asignaron aleatoriamente a 40 niños y adolescentes a una condición de TCC grupal $(\mathrm{n}=20)$ o a un grupo de tratamiento con sertralina ( $\mathrm{n}$ =20), ambos llevados a cabo durante 12 semanas con el objetivo de comparar la eficacia de ambos tratamientos a corto y largo plazo. Los sujetos tenían edades comprendidas entre los 9 y los 17 años $(M=8.9$ años) y ninguno había recibido tratamiento previamente para el TOC ni se encontraba recibiéndolo durante el estudio. La intervención cognitivoconductual consistió en 12 sesiones grupales (6-7 sujetos) de 90 minutos administradas de forma semanal. El programa fue adaptado del manual de March y Mulle (1998) incluyéndose en este caso los componentes de psicoeducación, entrenamiento cognitivo, EPR, sesiones familiares y prevención de recaídas. Los padres asistieron a los 15 minutos finales de cada sesión. Por otro lado, el tratamiento con sertralina fue administrado en dosis de 25 a $200 \mathrm{mg}$./día, siendo la dosis media administrada a lo largo del estudio de $137.5 \mathrm{mg}$. diarios. Los resultados mostraron que ambos tratamientos produjeron mejoras significativas respecto al postest en to- das las medidas excepto en depresión en la cual sólo se observaron diferencias significativas en el grupo sertralina. En cuanto a la comparación intergrupos, se observó que los tratados con TCC grupal mostraron un porcentaje significativamente inferior de recaídas que los sujetos que recibieron sertralina $(53 \%$ frente a $50 \%)$.

Se han realizando intentos por evaluar otro tipo de intervenciones de corte más cognitivo y comparar su eficacia con EPR. La terapia Metacognitiva (MTC) desarrollada por Wells (1997), según la cual los sujetos con TOC realizan interpretaciones metacognitivas erróneas de sus pensamientos intrusivos que conllevan una serie de evaluaciones erróneas como la fusión pensamiento-acción (los pensamientos son equivalentes a acciones, sucesos o intenciones) y a una serie de estrategias metacognitivas inadecuadas para controlar el pensamiento como la atención selectiva o la supresión del pensamiento que no hacen si no aumentar la conciencia del sujeto acerca de estas intrusiones (Simons, Scheider y Herpertz-Dahlmann, 2006). A diferencia de otras terapias cognitivas en las que se pretende modificar el contenido de las creencias y pensamientos, la terapia Metacognitiva tiene como objetivo cambiar las estrategias y evaluaciones que realiza el sujeto acerca de sus pensamientos. Simons et al. (2006) compararon los resultados obtenidos mediante la aplicación de la esta terapia con los observados mediante el uso de EPR en solitario en una muestra de 10 niños y adolescentes con TOC entre 8 y 17 años. Se programaron 20 sesiones para ambos tipos de intervención, con una frecuencia semanal. En la mayoría de los casos los padres podían acudir a las sesiones a excepción de que el paciente se negara a ello. El tratamiento con EPR se basó en el manual de March y Mulle (1998) aunque no incluyó técnicas de entrenamiento en el manejo de la ansiedad. Los resultados informaron de que ambos tratamientos se mostraron eficaces obteniendo mejoras significativas según sus puntuaciones en la CY-BOCS. Los beneficios se mantuvieron a los 3 y 24 meses de seguimiento. 
Posteriormente, Storch et al. (2007) han comparado los formatos intensivo y semanal de la TCC en un diseño de asignación aleatoria y utilizando una muestra mayor que la utilizada por Franklin et al. (1998), estudio en el que, como ya se ha comentado, los resultados sólo pudieron ser analizados a nivel descriptivo en esta variable debido al bajo tamaño muestral. Los participantes en el estudio fueron $40 \mathrm{ni}-$ ños y adolescentes entre 7 y 17 años $(M=13.3)$ con diagnóstico primario de TOC. Éstos fueron asignados de forma aleatoria a dos formatos de TCC, intensivo $(n=20)$ o semanal $(\mathrm{n}=20)$. El tratamiento estuvo basado en el manual de Lewin et al. (2005), ya comentado anteriormente, que incluye psicoeducación, entrenamiento cognitivo y EPR, aunque en este caso fue aplicado siguiendo un enfoque basado en la familia en el que al menos uno de los padres acudía a la sesión conjuntamente con el niño. En cuanto a los resultados, 18 sujetos completaron el tratamiento en el formato intensivo y 13 lo hicieron en el formato semanal. Ambos grupos mostraron reducciones significativas de las puntuaciones en CY-BOCS y en GCI-S del pretest al postest, las cuales fueron mantenidas en el seguimiento (3 meses), aunque no se obtuvieron diferencias significativas entre ambos grupos. Por último, tampoco se observaron diferencias significativas entre los sujetos que se encontraban tomando algún tipo de psicofármaco para el TOC durante el periodo del estudio y los que no lo hacían. Los autores concluyeron que ambos tratamientos resultaron eficaces y la utilización de uno u otro dependería de cuestiones de tiempo, severidad de la sintomatología, nivel de deterioro funcional, etc.

Con la finalidad de producir un aumento de la motivación para el tratamiento, Merlo et al. (2010) han evaluado la conveniencia de añadir la entrevista motivacional (EM) a la TCC. Para ello realizaron un estudio aleatorio en el que 16 niños y adolescentes $(\mathrm{M}=13.3$ años $)$ fueron asignados bien a TCC+EM o bien a TCC+Psicoeducación. Los resultados informaron que tras la cuarta sesión, la puntuación media en la CY-BOCS fue significativamente menor para el grupo EM que para el grupo Psicoeducación (también tras la sesión 9); asimismo también se produjo una reducción media significativamente mayor de la puntuación en esta misma escala en los sujetos que recibieron EM. De estos resultados se desprende que la entrevista motivacional puede ser un componente útil para acelerar los efectos producidos por la TCC en el tratamiento del TOC pediátrico. En la Tabla 2 se presentan los estudios descritos en este apartado.

\section{Diseños experimentales}

Son escasos los estudios experimentales acerca de la eficacia del tratamiento psicológico del TOC en niños y adolescentes. Hemos podido localizar cinco investigaciones hasta el momento que valoran el beneficio del tratamiento cognitivo-conductual frente a un grupo control. A pesar del bajo número de estudios experimentales, algunas variables han sido estudiadas de forma rigurosa mediante la utilización de grupos de control, tales como la comparación de la TCC frente a la medicación, el formato grupal versus individual, la utilización de EPR sin añadir otros componentes, la aplicación de la TCC en edad preescolar y la evaluación de la terapia de corte más cognitivo. Los grupos de control utilizados han sido del tipo lista de espera, píldora placebo y la atención psicológica.

Uno de los trabajos pioneros, fue el realizado por el equipo del Pediatric OCD Treatment Study (POTS, 2004). Se trata de uno de los estudios más completos y rigurosos ya que mediante la utilización de un diseño aleatorio de grupos paralelos se comparó la eficacia de 4 condiciones experimentales: (1) tratamiento con TCC, (2) tratamiento con sertralina (ISRS), (3) Tratamiento con TCC combinado con sertralina y (4) píldora placebo. Los participantes en el estudio fueron 112 niños y adolescentes entre 7 y 17 años con diagnostico primario de TOC. El tratamiento con TCC se llevó a cabo en 12 sesiones individuales de 1 hora a lo largo de 14 semanas y estuvo basado en el manual de March y Mulle (1998). El tratamiento con sertralina consistió en la administración de una dosis flexible entre 25 y $200 \mathrm{mg}$. / día durante 12 semanas. Los datos arrojados por este estudio aportan pruebas evidentes de los beneficios del uso de la TCC, sola o con medicación, para el tratamiento de niños y adolescentes que padecen el TOC. A pesar de que las diferencias no resultaron significativas entre los grupos tratados, la TCC se mostró superior a la medicación, arrojando un mayor porcentaje de remisión y un tamaño del efecto más elevado.

Barrett et al. (2004) compararon la eficacia de la TCC con implicación familiar en formato individual con el mismo tratamiento en formato grupal y con un grupo de control lista de espera. Los objetivos eran comprobar si el tratamiento con implicación familiar, además de disminuir la severidad del desorden, producía mejoras en el funcionamiento familiar (reducción del estrés familiar, reducción de la acomodación, etc.) y si el formato individual se mostraba más efectivo que el grupal. Para ello, setenta y siete jóvenes entre 7 y 17 años, con TOC, se asignaron de forma aleatoria a cada una de las tres condiciones, TCC familiar individual $(\mathrm{n}=24)$, TCC familiar grupal $(\mathrm{n}=29)$ y control de lista de espera ( $\mathrm{n}$ = 24). Para la aplicación del TCC con implicación familiar se utilizó el protocolo de tratamiento Freedom From Obsessions and Compulsions Using Cognitive-Behavioral Strategies (FOCUS) de Barrett y Farrell (en prensa), basado en el protocolo de TCC individual de March y Mulle (1998). Los resultados obtenidos tras el tratamiento indicaron que el $88 \%$ de los sujetos respondieron al mismo en la condición individual mientras que en la condición grupal lo hicieron el 76\%, según lo informado por los padres en la ADIS. Esta diferencia entre los dos grupos de tratamiento no resultó significativa, aunque ambos grupos sí se diferenciaron significativamente de la condición control, ya que ninguno de los participantes del grupo de lista de espera dejó de cumplir los criterios del TOC. Barrett, Farrell, Dadds y Boulter (2005) publican el seguimiento a los 12 y 18 meses de 48 de los participantes de ambos grupos de tratamiento, encontrando que en ambos momentos de evaluación, se encontraban libres de dia- 
gnóstico el $70 \%$ y el $84 \%$ de los participantes de la condición individual y grupal respectivamente, no resultando significativas estas diferencias. Asimismo, O'Leary, Barrett y Fjermestad (2009) presentan los datos del seguimiento de 19 de los participantes de la condición individual y del mismo número de la condición grupal, realizado a los 7 años de la finalización del tratamiento. Tras este periodo, el 79\% de la condición individual no cumplían los criterios diagnósticos para el TOC mientras que en la condición grupal no lo hacía el 95\%. Al igual que en momentos de evaluación anteriores, esta diferencia no resultó significativa. Estos estudios muestran que ambas modalidades de TCC familiar, grupal e individual, se muestran eficaces a corto y largo plazo en el tratamiento del TOC en la infancia y la adolescencia, observándose una tendencia a un mayor mantenimiento de las ganancias y una mayor reducción de la sintomatología depresiva en la condición grupal, avalando la conveniencia del uso de este formato de terapia ya que además presenta ventajas adicionales como la reducción de tiempo y costes, la provisión de apoyo social y modelado, etc.

Tabla 2. Diseños cuasi-experimentales con grupos de comparación.

\begin{tabular}{|c|c|c|c|c|c|c|c|c|}
\hline$\overline{\text { Estudio }}$ & Edad N & $\begin{array}{l}\text { N Criterio } \\
\text { Diagnostico }\end{array}$ & Diseño & Condiciones & $\begin{array}{l}\text { Otros } \\
\text { tratamien- } \\
\text { tos }\end{array}$ & $\begin{array}{l}\text { Medidas } \\
\text { Dependientes }\end{array}$ & $\begin{array}{l}\text { Reducción en la } \\
\text { sintomatología } \\
(\%)\end{array}$ & $\begin{array}{l}\text { Respuesta al tra- } \\
\text { tamiento } \\
(\%)\end{array}$ \\
\hline $\begin{array}{l}\text { Franklin et } \\
\text { al. (1998) }\end{array}$ & $\begin{array}{l}10- \\
17\end{array}$ & 14 DSM-III-R & $\begin{array}{l}\text { Ensayo no controla- } \\
\text { do con } 2 \text { grupos tra- } \\
\text { tados }\end{array}$ & $\begin{array}{l}\text { 1. EPR Intensi- } \\
\text { va } \\
\text { 2. EPR Semanal }\end{array}$ & $\begin{array}{l}\text { Medicación } \\
(57 \%)\end{array}$ & CY-BOCS & $\begin{array}{l}\text { Postest } \\
70 \text { Intensivo } \\
64 \text { Semanal } \\
\text { Seguimiento } \\
58 \text { Intensivo } \\
67 \text { Semanal } \\
\text { (CY-BOCS) }\end{array}$ & $\begin{array}{l}\text { Postest } \\
86 \\
\text { Seguimiento } \\
83 \\
\text { (cambio }>50 \% \\
\text { en CY-BOCS) }\end{array}$ \\
\hline $\begin{array}{l}\text { de Haan et } \\
\text { al. (1998) }\end{array}$ & $8-18 \quad 2$ & 22 DSM-III-R & $\begin{array}{l}\text { Ensayo no controla- } \\
\text { do aleatorio con } 2 \\
\text { grupos tratados }\end{array}$ & $\begin{array}{l}\text { 1. TCC indivi- } \\
\text { dual } \\
\text { 2. Clomiprami- } \\
\text { na }\end{array}$ & Ninguno & $\begin{array}{l}\text { CY-BOCS } \\
\text { LOI-CV }\end{array}$ & $\begin{array}{l}\text { EPR } \\
59.9 \\
\text { Clomipramina } \\
33.4 \\
\text { (CY-BOCS) }\end{array}$ & $\begin{array}{l}\text { EPR } \\
66.7 \\
\text { Clomipramina } \\
50 \\
\text { (Cambio } \geq 30 \% \\
\text { en CY-BOCS) }\end{array}$ \\
\hline $\begin{array}{l}\text { Ramos- } \\
\text { Asbarh } \\
\text { et al. } \\
(2005)\end{array}$ & $9-17 \overline{4}$ & 40 DSM-IV & $\begin{array}{l}\text { Ensayo no controla- } \\
\text { do aleatorio con } 2 \\
\text { grupos tratados }\end{array}$ & $\begin{array}{l}\text { 1. TCC Grupal } \\
\text { 2. Sertralina }\end{array}$ & Ninguno & $\begin{array}{l}\text { CY-BOCS } \\
\text { NIMH-OCS } \\
\text { CGI }\end{array}$ & No informa & $\begin{array}{l}\text { TCC Grupal } \\
95 \\
\text { Sertralina } \\
95 \\
\text { (Cambio } \geq 25 \% \\
\text { en CY-BOCS) }\end{array}$ \\
\hline $\begin{array}{l}\text { Simons et } \\
\text { al. (2006) }\end{array}$ & $8-17 \quad 1$ & $\begin{array}{l}10 \text { DSM-IV } \\
\text { CIE-10 }\end{array}$ & $\begin{array}{l}\text { Ensayo no controla- } \\
\text { do aleatorio con } 2 \\
\text { grupos tratados }\end{array}$ & $\begin{array}{l}\text { 1. MTC } \\
\text { 2. EPR }\end{array}$ & Ninguno & CY-BOCS & $\begin{array}{l}\text { EPR } \\
89,6 \text { (CY-BOCS) } \\
\text { MTC } \\
75 \text { (CY-BOCS) }\end{array}$ & $\begin{array}{l}100 \\
\text { (Cambio } \geq 30 \% \\
\text { en CY-BOCS) }\end{array}$ \\
\hline $\begin{array}{l}\text { Storch et } \\
\text { al. (2007) }\end{array}$ & $7-17 \quad 4$ & 40 DSM-IV-TR & $\begin{array}{l}\text { Ensayo no controla- } \\
\text { do aleatorio con } 2 \\
\text { grupos tratados }\end{array}$ & $\begin{array}{l}\text { 1.TCC Intensi- } \\
\text { vo } \\
\text { 2.TCC Semanal }\end{array}$ & $\begin{array}{l}\text { Medicación } \\
(60 \%)\end{array}$ & $\begin{array}{l}\text { CY-BOCS } \\
\text { CGI-S/I }\end{array}$ & $\begin{array}{l}\text { Postest } \\
63.3 \text { intensivo } \\
49.6 \text { semanal } \\
\text { Seguimiento } \\
60.6 \text { intensivo } \\
\text { 61.4 semanal } \\
\text { (CY-BOCS) }\end{array}$ & $\begin{array}{l}\text { Postest } \\
90 \text { intensivo } \\
65 \text { semanal } \\
\text { Seguimiento } \\
75 \text { intensivo } \\
50 \text { semanal } \\
\\
(\text { CGI-I } \geq 6)\end{array}$ \\
\hline $\begin{array}{l}\text { Merlo et al. } \\
(2010)\end{array}$ & $6-17 \quad 1$ & 16 DSM-IV & $\begin{array}{l}\text { Ensayo no controla- } \\
\text { do aleatorio con } 2 \\
\text { grupos tratados }\end{array}$ & $\begin{array}{l}\text { 1. TCC intensi- } \\
\text { vo+ EM } \\
\text { 2. TCC intensi- } \\
\text { vo+ PE }\end{array}$ & No informa & $\begin{array}{l}\text { ADIS-CP } \\
\text { CY-BOCS } \\
\text { CGI }\end{array}$ & $\begin{array}{l}\text { 69.7 TCC+EM } \\
54.8 \text { TCC+EM } \\
(\mathrm{CY}-\mathrm{BOCS})\end{array}$ & No informa \\
\hline
\end{tabular}

ADIS: Protocolo de Entrevista de Trastornos de Ansiedad del DSM; CY-BOCS: Escala Yale-Brown de Obsesiones y Compulsiones para Niños; LOI-CV: Inventario de Obsesiones de Leyton; CGI: Escala de Impresión Clínica Global; NIMH: Entrevista del National Institute of Mental Health Diagnostic for Children; TCC: Terapia cognitivo-conductual; EPR: Exposición con prevención de respuesta; EM: Entrevista motivacional; MTC: Terapia metacognitiva. PE: Psicoeducación.

Bolton y Perrin (2008) han evaluado la eficacia, frente a un grupo control, de la aplicación de la EPR a niños y adolescentes con TOC. Los participantes en el estudio fueron 20 sujetos entre 7 y 17 años $(M=13.2)$. Éstos fueron aleatoriamente asignados a un grupo de tratamiento con EPR ( $\mathrm{n}=$
10) o a un grupo control de lista de espera $(n=10)$. Los autores concluyeron que la EPR en solitario es un tratamiento eficaz para reducir la sintomatología del TOC infantil, destacando las ventajas de este procedimiento, tales como la sencillez en la aplicación, el ahorro de tiempo, etc. 
Freeman et al. (2008) compararon la eficacia de la TCC familiar para el TOC en niños de edades muy tempranas. Para ello, utilizaron un diseño controlado aleatorio en el que asignaron a 42 niños entre 4 y 8 años $(M=7.11)$ a un grupo de tratamiento con TCC familiar $(\mathrm{N}=22)$ o a un grupo de placebo psicológico de entrenamiento en relajación $(\mathrm{N}=$ 20). Ambos grupos de intervención siguieron un protocolo compuesto por 12 sesiones a lo largo de 14 semanas, incluyendo a niños y padres, excepto las dos primeras sesiones a las que sólo acudieron los padres. El programa cognitivoconductual familiar utilizado (Choate-Summers et al., 2008) incluyó adaptaciones a los niveles de desarrollo de los niños en cuanto a sus habilidades cognitivas y emocionales así como al funcionamiento familiar típico a estas edades. El grupo de atención psicológica placebo recibió un programa de relajación compuesto por los siguientes elementos: (1) Psicoeducación afectiva: identificación de sentimientos y emociones, estrés y ansiedad, (2) entrenamiento en relajación muscular progresiva, y (3) Programa de refuerzo. Los datos reflejaron que la TCC se mostró más eficaz que la relajación.

El último estudio experimental realizado en este ámbito, pone a prueba una TCC esencialmente cognitiva frente a un grupo control de lista de espera, ya que este tipo de terapia no había sido evaluada hasta el momento de forma experimental para el tratamiento del TOC en jóvenes. Se trata del trabajo realizado por Williams et al. (2010) en el que 21 sujetos entre 9 y 18 años fueron distribuidos de forma aleatoria a una TCC centrada en las cogniciones disfuncionales, o a un grupo control de lista de espera. El programa cognitivo conductual estuvo basado en el trabajo realizado por Salkovskis (1998). El tratamiento se compuso de 10 sesiones que incluían el componente cognitivo junto a ejercicios de exposición orientados a poner a prueba las cogniciones más que a la habituación del malestar, y psicoeducación acerca de la ansiedad. El grupo tratado experimentó una mejora significativamente mayor que el grupo lista de espera. En la Tabla 3 se presentan los estudios descritos en este apartado.

\section{Discusión}

En lo que se refiere a los diseños pre-postest de un solo grupo, los primeros informes de los que se ha tenido noticia surgen a principios de 1980, habiéndose realizado numerosos estudios de este tipo hasta la actualidad. En cuanto a las intervenciones examinadas, éstas se caracterizan por ser homogéneas para todos los participantes, con la excepción del estudio de Bolton, Collins y Steinberg (1983). Los tratamientos aplicados son, en la mayoría de los casos, programas multicomponente, compuestos, al igual que en los estudios de caso y caso único, por EPR y técnicas cognitivas, en los cuales la familia es implicada en mayor o menor nivel según el protocolo seguido o la edad de los sujetos. Sin embargo, también encontramos evaluaciones de programas esencialmente conductuales, compuestos sólo por EPR (Apter et al., 1984; Bolton et al., 1983, Scahill et al., 1996) y programas puramente cognitivos (Williams et al., 2002). Los protocolos o manuales, seguidos en la aplicación de las intervenciones son los de March y Mulle (1998), Lewin et al. (2005), Barrett (2009), Piacentini et al. (1998), Schwartz (1996), Wells (1997) y Salkovskis (1998). En cuanto a las modalidades de aplicación además de forma clásica de TCC individual semanal, encontramos la evaluación de formatos de aplicación grupal (Fischer et al., 1998, Martin y Thienemann, 2005, Thienemann et al., 2001) intensivo (Storch et al., 2006) y telefónico (Turner et al., 2009). Asimismo también se presta atención al contexto de aplicación de la TCC, destacando los estudios realizados en ámbito del régimen hospitalario (Bjorgvinsson et al., 2008; Bolton et al., 1983) y los llevados a cabo en clínicas comunitarias no académicas (Farrell et al., 2010; Valderhaug et al., 2007) que suponen la evaluación de la efectividad de los programas. Por otro lado, en relación a las muestras utilizadas éstas incluyeron entre 6 y 92 sujetos, los cuales tuvieron edades comprendidas entre los 3 y los 18 años, los criterios diagnósticos del TOC utilizados fueron los del DSM-III-R, DSM-IV y CIE-10. En cuanto a la toma de fármacos por parte de los sujetos que participaron en los ensayos, tan solo 1 de los 17 estudios informa de que éstos se encontraban totalmente libres de la toma de medicación para el TOC (Benazon et al., 2002), variando el porcentaje de los sujetos medicados entre el 8.3 y el $93 \%$ en los restantes estudios, lo que hace difícil atribuir los resultados de eficacia informados, en exclusiva a la TCC. Teniendo esto en cuenta, el porcentaje de sujetos que respondieron al tratamiento según diferentes criterios utilizados en cada uno de los estudios, varía entre el 50 y el $91,7 \%$ en el postest. Asimismo los pocos estudios que aportan medidas de seguimiento informan de cambios en el 50-100\% de los participantes (Storch et al., 2006; Valderhaug et al., 2007). En este sentido, la reducción de la sintomatología según los datos informados arroja porcentajes entre el 25 y el $68 \%$ en el postest y el 46 y el $70 \%$ en el seguimiento. Las principales medidas de mejora utilizadas fueron instrumentos psicométricos estandarizados como la CY-BOCS, CGI, NIMH y ADIS, desapareciendo casi por completo las medidas observacionales en este tipo de diseños, ya que sólo se contemplaron como criterio de respuesta en un caso (Bolton et al., 1983).

El siguiente grupo de estudios revisado, se compone de los ensayos que utilizaron un diseño cuasi-experimental de comparación de grupos de tratamiento. En todos los casos se compararon dos grupos de tratamiento, a los cuales los sujetos fueron asignados de forma aleatoria, excepto en uno de los estudios (Franklin et al., 1998). Los tamaños muestrales en los ensayos variaron entre 10 y 40 sujetos, con edades comprendidas entre los 7 y los 18 años. Las comparaciones realizadas son por un lado, entre dos tratamientos psicológicos ya sea diferenciándose en el tipo de técnica cognitivoconductual utilizada, terapia metacognitiva vs. EPR (Simons et al., 2006), en el formato de aplicación de la TCC, intensivo vs. semanal (Franklin et al., 1998; Storch et al., 2007) o en el cambio de uno de los componentes del programa, TCC 
Tabla 3. Diseños experimentales.

\begin{tabular}{|c|c|c|c|c|c|c|c|c|}
\hline Estadio & Edad N & $\begin{array}{l}\text { Criterio } \\
\text { Diagnóstico }\end{array}$ & Diseño & Condiciones & $\begin{array}{l}\text { Otros } \\
\text { tratamientos }\end{array}$ & $\begin{array}{l}\text { Medidas } \\
\text { Dependientes }\end{array}$ & $\begin{array}{l}\text { Reducción en la } \\
\text { sintomatología } \\
(\%)\end{array}$ & $\begin{array}{l}\text { Respuesta al } \\
\text { tratamiento }(\%)\end{array}$ \\
\hline $\begin{array}{l}\text { POTS } \\
(2004)\end{array}$ & $\begin{array}{ll}7-17 & 112\end{array}$ & DSM-IV & $\begin{array}{l}\text { Estudio con- } \\
\text { trolado } \\
\text { aleatorio con } 4 \\
\text { grupos de } \\
\text { comparación }\end{array}$ & $\begin{array}{l}\text { 1. TCC } \\
\text { 2. Sertralina } \\
\text { 3. TCC+Sertralina } \\
\text { 4. Placebo }\end{array}$ & Ninguno & $\begin{array}{l}\text { ADIS-C } \\
\text { CY-BOCS }\end{array}$ & $\begin{array}{l}\text { TCC } \\
46.2 \\
\text { Sertralina } \\
30 \\
\text { TCC+Sertralina } \\
50 \\
\text { Placebo } \\
14.7 \\
\text { (CY-BOCS) }\end{array}$ & $\begin{array}{l}\text { TCC } \\
39.3 \\
\text { Sertralina } \\
21.4 \\
\text { TCC+Sertralina } \\
53.6 \\
\text { Placebo } \\
3.6 \\
\text { (Cambio } \geq 10 \% \\
\text { en CY-BOCS) } \\
\end{array}$ \\
\hline $\begin{array}{l}\text { Barrett et } \\
\text { al. (2004) }\end{array}$ & $7-17 \quad 77$ & DSM-IV & $\begin{array}{l}\text { Estudio con- } \\
\text { trolado } \\
\text { aleatorio con } 3 \\
\text { grupos de } \\
\text { comparación }\end{array}$ & $\begin{array}{l}\text { 1. TCC Familiar } \\
\text { Individual } \\
\text { 2. TCC Familiar } \\
\text { Grupal } \\
\text { 3. Lista de espera }\end{array}$ & $\begin{array}{l}\text { Medicación } \\
\text { - TCC Fami- } \\
\text { liar Individual } \\
(12.5 \%) \\
\text { - TCC Fami- } \\
\text { liar Grupal } \\
(31 \%) \\
\text { - Lista de es- } \\
\text { pera }(20.83 \%)\end{array}$ & $\begin{array}{l}\text { ADIS } \\
\text { CY-BOCS } \\
\text { NIMH-OCS } \\
\text { CGAS }\end{array}$ & $\begin{array}{l}\text { TCC Familiar In- } \\
\text { dividual } \\
65 \\
\text { TCC Familiar } \\
\text { Grupal } \\
61 \\
\\
\text { (CY-BOCS) } \\
\end{array}$ & $\begin{array}{l}\text { TCC Familiar In- } \\
\text { dividual } \\
88 \\
\text { TCC Familiar } \\
\text { Grupal } \\
76 \\
\text { Lista de espera } \\
0 \\
\text { (Remisión ADIS) } \\
\end{array}$ \\
\hline $\begin{array}{l}\text { Bolton y } \\
\text { Perrin } \\
(2008)\end{array}$ & $7-17 \quad 20$ & DSM-IV & $\begin{array}{l}\text { Estudio con- } \\
\text { trolado } \\
\text { aleatorio con } 2 \\
\text { grupos de } \\
\text { comparación }\end{array}$ & $\begin{array}{l}\text { 1.EPR } \\
\text { 2. Lista de espera }\end{array}$ & Ninguno & $\begin{array}{l}\text { ADIS-C/P } \\
\text { CY-BOCS } \\
\text { CHOCI }\end{array}$ & $\begin{array}{l}\text { EPR } \\
42 \\
\text { Lista de espera } \\
1.8 \\
\text { (CY-BOCS) }\end{array}$ & $\begin{array}{l}\text { (Cambios signifi- } \\
\text { cativos en CY- } \\
\text { BOCS) } \\
\end{array}$ \\
\hline $\begin{array}{l}\text { Freeman } \\
\text { et al. } \\
\text { (2008) }\end{array}$ & $4-8 \quad 42$ & DSM-IV & $\begin{array}{l}\text { Estudio con- } \\
\text { trolado } \\
\text { aleatorio con } 2 \\
\text { grupos de } \\
\text { comparación }\end{array}$ & $\begin{array}{l}\text { 1. TCC Familiar } \\
\text { 2. Entrenamiento } \\
\text { en relajación (con- } \\
\text { trol) }\end{array}$ & $\begin{array}{l}\text { ISRS } \\
(14.3)\end{array}$ & $\begin{array}{l}\text { CY-BOCS } \\
\text { CGI-I }\end{array}$ & $\begin{array}{l}\text { TCC Familiar } \\
37 \\
\text { Entrenamiento en } \\
\text { relajación } \\
21.2 \\
\text { (CY-BOCS) }\end{array}$ & $\begin{array}{l}\text { TCC-Familiar } \\
50 \\
2 . \text { Entrenamiento } \\
\text { en relajación } \\
20 \\
(\text { CGI-I } \geq 6)\end{array}$ \\
\hline $\begin{array}{l}\text { Williams } \\
\text { et al. } \\
\text { (2010) }\end{array}$ & $9-18 \quad 21$ & DSM-IV & $\begin{array}{l}\text { Estudio con- } \\
\text { trolado } \\
\text { aleatorio con } 2 \\
\text { grupos de } \\
\text { comparación } \\
\end{array}$ & $\begin{array}{l}\text { 1. Terapia Cogni- } \\
\text { tiva } \\
\text { 2. Lista de Espera }\end{array}$ & $\begin{array}{l}\text { Medicación } \\
(33 \%)\end{array}$ & CY-BOCS & $\begin{array}{l}\text { Terapia Cognitiva } \\
47.6 \\
\text { Lista de Espera } \\
7 \\
\text { (CY-BOCS) }\end{array}$ & No informa \\
\hline
\end{tabular}

ADIS: Protocolo de Entrevista de Trastornos de Ansiedad del DSM; CY-BOCS: Escala Yale-Brown de Obsesiones y Compulsiones para Niños; CGI: Escala de Impresión Clínica Global; NIMH: Entrevista del National Institute of Mental Health Diagnostic for Children; CHOCI: Inventario Obsesivo Compulsivo para Niños; TCC: Terapia cognitivo-conductual; EPR: Exposición con prevención de respuesta.

intensivo con entrevista motivacional (EM) vs. TCC intensivo junto a psicoeducación (Merlo et al., 2010). Por otro lado, encontramos la comparación entre el tratamiento psicológico y el tratamiento farmacológico, en los que se ha estudiado la eficacia diferencial de la EPR frente a la clomipramina y de la TCC grupal frente a la sertralina (de Haan et al., 1998; Ramos-Asbahr et al., 2005). Los programas seguidos para la aplicación de los tratamientos psicológicos han sido los de Lewin et al. (2005), Wells (1997) y March y Mulle (1998). Las dosis medias de fármacos administradas fueron de 112. mg./día para la clomipramina y de 137.5 miligramos diarios para la sertralina. En cuanto a los resultados de eficacia, el porcentaje de sujetos que respondieron a los tratamientos psicológicos varió entre el 65 y el 100\%, con una reducción de los síntomas entre el 50 y el 90\% en el post-test. Los datos de seguimiento aportados por dos de los estudios informan de porcentajes de respuesta entre 50 y el $83 \%$, con porcentajes de mejora del TOC entre 60 y el 67\% (Franklin et al., 1998; Storch et al., 2007). Los porcentajes para el tratamiento farmacológico fueron del $50-95 \%$ y del $33.4 \%$ para respuesta al tratamiento y reducción de la sintomatología respectivamente. Las medidas en las que se establecieron tanto los criterios de mejora como los porcentajes de cambio fueron CY-BOCS y CGI.

Por último, los diseños experimentales que incorporan grupo de control y asignación aleatoria de los participantes a las diferentes condiciones evaluadas, comenzaron a realizarse en el año 2004, siendo el último estudio llevado a cabo recien- 
temente en 2010. El número de condiciones experimentales varía entre 2 y 4, según el estudio. Las muestras utilizadas se compusieron de un número de sujetos entre 20 y 112, los cuales presentaron edades comprendidas entre los 4 y los 18 años. El criterio diagnóstico utilizado ha sido el DSM-IV en todos los casos. Los tratamientos evaluados frente a un grupo de control han sido protocolos de tratamiento multicomponente de TCC individual (POTS, 2004), TCC familiar tanto en formato individual como grupal (Barrett et al., 2004; Freeman et al., 2008), programas de EPR en solitario (Bolton y Perrin, 2008) y de terapia cognitiva (Williams et al., 2010). Asimismo, se ha puesto a prueba la eficacia del tratamiento combinado de TCC individual junto a sertralina (POTS, 2004). Las condiciones de control utilizadas han sido tanto de tipo activo, atención psicológica (Freeman et al., 2008) o placebo (POST, 2004), como de tipo inactivo, grupo de lista de espera (Barrett et al., 2004; Bolton y Perrin, 2008; Williams et al., 2010). Los protocolos en los que se han basado los tratamientos han sido los de March y Mulle (1998), Barrett y Farrell (en prensa), Choate-Summers et al. (2008) y Salkovskis (1999). En tres de los estudios mencionados, una parte de los sujetos también se encontraban tomando algún tipo de ISRS para el tratamiento del TOC, en concreto los datos informan de porcentajes entre 12.5 y $33 \%$ de sujetos medicados (Barrett et al., 2004, Freeman et al., 2008, Williams et al., 2010). Este hecho debe ser tenido en cuenta a la hora de interpretar los resultados obtenidos en cuanto a la eficacia, los cuales, han informado de porcentajes de respuesta al tratamiento psicológico entre el 40 y el $88 \%$, mientras que para el tratamiento combinado la respuesta fue del $53.6 \%$. Respecto a la mejora del desorden los porcentajes de reducción de síntomas varían entre el 37 y el $48 \%$ para el tratamiento psicológico y el $50 \%$ para el tratamiento combinado. No se dispone de datos de seguimiento en los que se mantengan las condiciones de control. Las medidas de resultado en este caso también son los instrumentos de evaluación validados CY-BOCS, CGI y ADIS.

\section{Conclusiones y perspectivas de futuro}

Como una de las conclusiones principales, hemos de indicar que el componente principal del tratamiento del TOC infantil y adolescente es la exposición con prevención de respuesta, procedimiento que, tal y como se ha comprobado recientemente, proporciona beneficios similares utilizado bien solo o en combinación con otras técnicas (Rosa-Alcázar et al., 2008). Sin embargo, en muchas ocasiones, las intervenciones llevadas a cabo en el TOC infantil han incluido otros componentes además de la exposición con prevención de respuesta, como son psicoeducación neurobiológica, el entrenamiento a los padres o intervenciones basadas en la familia y técnicas cognitivas y de manejo de ansiedad.

En cuanto a la modalidad de aplicación, comprobamos que tanto el formato individual como grupal han sido puestos a prueba en numerosos ensayos abiertos y un estudio controlado, siendo la modalidad grupal menos estudiada. Se han encontrado porcentajes de mejora del trastorno entre el 25 y el $60 \%$ para el tratamiento en grupo, mientras que para la TCC individual éstos han variado entre el entre el $30 \mathrm{y}$ el $65 \%$, no siendo significativas las diferencias entre ambos formatos. Por lo tanto, la elección de uno u otro deberá basarse en las necesidades y características del paciente así como en el contexto de aplicación de la TCC.

Otra característica importante de los tratamientos es el nivel de intensidad con el que pueden ser aplicados. En este aspecto, se distingue entre la terapia semanal, administrada a razón de una sesión a la semana, alcanzando las 12 sesiones en un periodo de dos meses y la terapia intensiva, en la cual se reciben entre 4 y 5 sesiones semanales por lo que el tratamiento tiene una duración menor en el tiempo. En este caso, tampoco se han encontrado diferencias significativas entre ambas formas, en los estudios de comparación realizados al respecto. No obstante, los expertos recomiendan el formato intensivo cuando lo que prima es la rapidez de actuación, ya sea porque el niño presente un elevado deterioro funcional o por la necesidad de desplazamiento geográfico para recibir la intervención, entre otros motivos (March et al., 1997).

En cuanto a la aplicación de la TCC en formatos novedosos que implican el uso de medios de comunicación, tales como el contacto telefónico, los resultados obtenidos fueron similares a los de otros estudios con el tratamiento clásico, encontrándose porcentajes de mejora y respuesta medios y medios altos, lo que indica que podría tratarse de una opción interesante en ciertos casos.

Otro de los aspectos relevantes cuando se trata de intervenir en el TOC de niños y adolescentes es el grado de implicación de la familia en el proceso de tratamiento. La familia influye en el trastorno modificando. En ocasiones, el curso del TOC influye en la vida, actividades, interacciones y afectos de la familia (March, 1995). Esta influencia mutua queda reflejada en el desarrollo por parte de la familia de creencias, actitudes y conductas relacionadas con el trastorno, que pueden situarse tanto en el extremo de la acomodación, la implicación y la colaboración en los síntomas, como en el polo de la ira, la culpabilización y el rechazo al niño. Por otro lado, otro de los roles que los padres suelen adquirir en la intervención, es el de la actuación como coterapeutas. Para ello, éstos son entrenados por el clínico para la realizar tareas como la guía de los ejercicios de exposición con prevención de respuesta en el hogar, la aplicación de programas de refuerzo, el manejo del estrés y la frustración del niño, etc. Asimismo señalar que, por lo general, la implicación de la familia aumenta conforme disminuye la edad del niño.

Podemos concluir resaltando el ingente desarrollo de la investigación en este ámbito experimentado en los últimos años, además de la clara evidencia a favor de la eficacia del tratamiento cognitivo-conductual para el niño y adolescente con TOC, evaluado mediante la utilización de los diferentes tipos de diseños de investigación así como su aplicación en distintos formatos (intensivo/semanal, individual/grupal, con/sin implicación familiar, etc.). Sin embargo, también 
debemos hacer mención a las deficiencias observadas de cara a poder subsanarlas en futuros estudios, como son la baja representación de estudios experimentales, la escasez de medidas de seguimiento a medio y largo plazo, la falta de utilización de medidas observacionales y el escaso control de la sintomatología encubierta y trastornos comórbidos.

\section{Referencias}

Abramowitz, J. S., Whiteside, S. P., y Deacon, B. J. (2005). The effectiveness of treatment for pediatric obsessive-compulsive disorder: A metaanalysis. Behavior Therapy, 36, 55-63.

American Psychiatric Association. (2000). Diagnostic and statistical manual of mental disorders (DSM-IV-TR). Washington, D.C.: American Psychiatric Association.

Apter, A., Bemhout, E. y Tyrano, S. (1984). Severe obsessive compulsive disorder in adolescence: A report of eight cases. Journal of Adolescence, 7 , 349-358.

Barrett, P. (2009). FOCUS for Life: Freedom from obsessions and compulsions using skills. Therapist Manual and Workbooks. Disponible en Brisbane, Queensland: Pathways Health and Research Centre.

Barrett, P. M., Farrell, L., y March, J. S. (2004). Cognitive-behavioral family treatment of childhood obsessive compulsive disorder: A controlled trial. Journal of the American Academy of Child and Adolescent Psychiatry, 43, 46-62.

Barrett, P. M., Farrell, L., Dadds, M. y Boulter, N. (2005). Cognitivebehavioral family treatment of childhood obsessive-compulsive disorder: Long-term follow-up and predictors of outcome. Journal of the American Academy of Child and Adolescent Psychiatry, 44, 1005-1014.

Barrett, P.M. y Farrell, L. (en prensa). FOCUS: Freedom from Obsessions and Compulsions Using Cognitive-Behavioural Strategies. Australian Academic Press.

Benazon, N.R., Ager, J. y Rosenberg, D.R. (2002). Cognitive behavior therapy in treatment -naive children and adolescents with obsessivecompulsive disorder: an open trial. Behaviour Research and Therapy, 40, 529-539.

Bjorgvinsson, T., Wetterneck, C.T., Powell, D.M., Chasson, G.S, Webb, S.A, Hart, J., Heffelfinger, S., Azzouz, R., Entricht, T.L., Davidson, J.E. y Stanley, M.A. (2008). Treatment outcome for adolescent obsessivecompulsive disorder in a specialized hospital setting. Journal of Psychiatric Practice, 14, 137-145.

Bolton, D., Collins, S. y Steinberg, D. (1983). The treatment of obsessivecompulsive disorder in adolescence: A report of 15 cases. British Journal of Psychiatry, 142, 456-464.

Bolton, D., Luckie, M. y Steimberg, D. (1995). Long-term course of obsessive compulsive disorder treated in adolescence. Journal of the American Academy of Child and Adolescent Psychiatry, 34, 1441-1450.

Bolton, D. y Perrin, S. (2008). Evaluation of exposure with responseprevention for obsessive compulsive disorder in childhood and adolescence. Journal of Behavior Therapy

Canals, J., Doménech, E., Carbajo, G. y Blade, J. (1997). Prevalence of DSM-III-R and ICD-10 psychiatric disorders in a Spanish population of 18-year-olds. Acta Psychiatrica Scandinavica, 96, 287-94.

Choate-Summers, M.L., Freeman, J.B., Garcia, A.M., Coyne, L., Przeworski, A. y Leonard, H.L. (2008). Clinical considerations when tailoring cognitive behavioral treatment for young children with obsessive compulsive disorder. Education and Treatment of Children, 31, 395-416.

Clark, D. (1982). Primary obsessional slowness: a nursing treatment programme with a 13 year old male adolescent. Behaviour Research and Therapy, 20, 289-292.

de Haan, E., Hoogduin, K., Buitelaar, J., y Keijsers, G. (1998). Behavior therapy versus clomipramine for treatment of obsessive-compulsive disorder in children and adolescents. Journal of the American Academy of Child and Adolescent Psychiatry, 37, 1022-1029.

Farrell, L.J., Schlup, B. y Boschen, M.J. (2010). Cognitive-behavioral treatment of childhood obsessive-compulsive disorder in community-based clinical practice: clinical significance and benchmarking against efficacy. Behaviour Research and Therapy, 48, 409-417.
Nota: Este trabajo es resultado del proyecto de investigación 08703/PHCS/08 financiado con cargo al Programa de Generación de Conocimiento Científico de Excelencia de la Fundación SénecaAgencia de Ciencia y Tecnología de la Región de Murcia (España) en el marco del II PCTRM 2007-10.

Fischer, D.J., Himle, J. A. y Hanna, G.L. (1998). Group behavioural therapy for adolescents with obsessive-compulsive disorder: Preliminary outcomes. Research on Social Work Practice, 8, 629-636.

Franklin, M.E., Kozak, M.J., Cashman, L., Coles, M., Rheingold, A. y Foa, E.B. (1998). Cognitive-behavioral treatment of pediatric obsessivecompulsive disorder: An open clinical trial. Journal of the American Academy of Child and Adolescent Psychiatry, 37, 412-419.

Freeman, J.B, Garcia, A.M, Coyne, L., Ale, Ch., Przeworski, A., Himle, M., Compton, S. y Leonard, H.L. (2008). Early childhood OCD: Preliminary findings from a family-based cognitive-behavioral approach. Journal of the American Academy of Child and Adolescent Psychiatry, 47, 593-602.

Fullana, M.A._Mataix-Cols, D., Caspi, A., Harrington, B.A., Grisham, J., Moffitt, T., y Poulton, R. (2009). Obsessions and compulsions in the community: prevalence, interference, help-seeking, developmental stability and cooccurring psychiatric conditions. American. Journal Psychiatry, 166, 329-336.

Geller, D.A., Biederman, J., Stewart, S.E., Mullin, B., Martin, A., Spencer, T. y Faraone, S.V. (2003). Which SSRI? A meta-analysis of pharmacotherapy trials in pediatric obsessive-compulsive disorder. American Journal of Psychiatry, 160, 1919-1928.

Guy, W. (1976). Assessment manual for psychopharmacology, revised (DHEW Publication No. ABM 76-366). Washington, DC: US Government Printing Office.

Himle, J.A., Fischer, D.J., Van Etten, M., Janeck, A. y Hanna, G.L. (2003). Group behavioral therapy for adolescents with tic-related and nonticrelated obsessive-compulsive disorder. Depression and Anxiety, 17, 73-77.

Lewin, A.B., Storch, E.A., Merlo, L.J., Murphy, T.K. y Geffken, G.R. (2005). Intensive cognitive behavioral therapy for pediatric obsessive compulsive disorder: a treatment protocol for mental health providers. Psychological Services, 2, 91-104.

March, J.S. (1995). Cognitive-behavioral psychotherapy for children and adolescents with OCD: a review and recommendations for treatment. Journal of the American Academy of Child and Adolescent Psychiatry, 34, 7-18.

March, J.S., Frances, A., Kahn, D.A. y Carpenter, D. (1997). The Expert Consensus Guidelines ${ }^{\circledR}$ : Treatment of Obsessive-Compulsive Disorder. Journal of Clinical Psychiatry, 58 (suppl 4).

March, J.S., Mulle, K., y Herbel, B. (1994). Behavioral psychotherapy for children and adolescents with obsessive-compulsive disorder: An open trial of a new protocol driven treatment package. Journal of the American Academy of Child and Adolescent Psychiatry, 33, 333-341.

March, J.S. y Mulle, K. (1998). OCD in children and adolescents: a cognitivebehavioral treatment manual. Nueva York: Guilford.

Martin, J. y Thienemann, M. (2005). Group cognitive-behavior therapy with family involvement for middle-school-age children with obsessivecompulsive disorder: A pilot study. Child Psychiatry and Human Development, 36, 113-124.

Merlo, L.J., Lehmkuhl, H.D., Geffken, G.R. y Storch, E.A. (2009). Decreased family accommodation associated with improved therapy outcome in pediatric obsessive-compulsive disorder. Journal of Consulting and Clinical Psychology, 77, 355-360.

Merlo, L.J., Storch, E.A., Lehmkuhl, H.D., Jacob, M.L., Murphy, T.K., Goodman, W. K. y Geffken, G. R. (2010). Cognitive behavioral therapy plus motivational interviewing improves outcome for pediatric obsessive-compulsive disorder: A preliminary study. Cognitive Behaviour Therapy, 39, 24-27.

National Institute of Mental Health. (1985). Rating scales and assessment instruments for use in pediatric psychopharmacology research. Psychopharmacological Bulletin, 21, 839-843. 
O'Kearney, R. (2007). Benefits of cognitive-behavioural therapy for children and youth with obsessive-compulsive disorder: re-examination of the evidence. Australian and New Zealand Journal of Psychiatry, 41, 199-212.

O'Leary, E.M., Barrett, P. y Fjermestad, K.W. (2009). Cognitive-behavioral family treatment for childhood obsessive-compulsive disorder: a 7-year follow-up study. Journal of Anxiety Disorders, 23, 973-978.

Pediatric OCD Treatment Study (POTS) Team. (2004). Cognitive-behavior therapy, sertraline, and their combination for children and adolescents with obsessive-compulsive disorder: The pediatric OCD treatment study (POTS) randomised controlled trial. Journal of the American Medical Association (JAMA), 292, 1969-1976.

Perlmutter, S.J., Leitman, S.F., Garvey, M.A., Hamburger, S., Feldman, E. y Leonard, H.L. (1999). Therapeutic plasma exchange and intravenous immunoglobulin for obsessive-compulsive disorder and tic disorders in childhood. The Lancet, 354, 1153-1158.

Piacentini, J., Bergman, R. L., Jacobs, C., McCracken, J. T., y Kretchman, J. (2002). Open trial of cognitive behaviour therapy for childhood obsessive-compulsive disorder. Journal of Anxiety Disorders, 16, 207-219

Piacentini, J., Jacobs, C., Maidment, K., y Bergman, R. L. (1998). Individual $C B T$ and family (ERP/family) treatment: A multicomponent treatment program for children and adolescents with obsessive-compulsive disorder. Manuscrito no publicado, Los Ángeles, Estados Unidos.

Piacentini, J. y Jaffer, M. (1999). Measuring functional impairment in youngsters with OCD: manual for the child OCD impact scale (COIS). UCLA Department of Psychiatry, Los Angeles.

Piacentini, J., Gitow, A., Jaffer, M., Graae, F. y Whitaker, A. (1994). Outpatient behavioural treatment of child and adolescent obsessivecompulsive disorder. Journal of Anxiety Disorders, 8, 277-289.

Ramos-Asbahr, F., Castillo, A.R., Ito, L.M., Latorre, M.R.D.O., Moreira, M.N., Lotufo-Neto, F. (2005). Group cognitive-behavioral therapy versus sertraline for the treatment of children and adolescents with obsessive-compulsive disorder. Journal of the American Academy of Child and Adolescent Psychiatry, 44, 1128-1136.

Rosa-Alcázar, A. I., Olivares P. e Iniesta, M. (2010). El tratamiento del trastorno obsesivo compulsivo (pp.120-151). En A. I. Rosa-Alcazar y J. Olivares (eds.), Tratamiento Psicológico del Trastorno Obsesivo Compulsivo en Niños y Adolescentes (pp. 175-215). Madrid: Pirámide.

Rosa-Alcázar, A., Sánchez-Meca, J., Gómez-Conesa, A. y Marín-Martínez, F. (2008). Psychological treatment of obsessive-compulsive disorder: A meta-analysis. Clinical Psychology Review, 28, 1310-1325.

Salkovskis, P.M. (1998). Psychological approaches to the understanding of obsessional problems. En R.P. Swinson, M.M. Antony, S.J. Rachman y M.A. Richter, (eds.), Obsessive-compulsive disorder: theory, research and treatment. Nueva York: Guilford.

Scahill, L., Riddle, M. A., McSwiggin-Hardin, M., Ort, S. I., King, R. A. Goodman, W. K., Cicchetti, D., y Leckman, J. F. (1997). Children's yale-brown obsessive compulsive scale: Reliability and validity. Journal of the American Academy of Child and Adolescent Psychiatry, 36, 844-852.

Scahill, L., Vitulano, L.A., Brenner, E.M., Lynch, K.A. y King, R.A. (1996). Behavioral therapy in children and adolescents with obsessivecompulsive disorder: A pilot study. Journal of Child and Adolescent Psychopharmacology, 6, 191-202.

Schwartz, J. M. (1996). Brain lock. Nueva York: Harper Collins.

Shaffer, D., Fisher, P., Lucas, C.P., Dulcan, M.K. y Schwab-Stone, M.E. (2000). NIMH Diagnostic Interview Schedule for Children Version IV (NIMH DICS-IV): description, differences from previous versions, and reliability of some common diagnoses. Journal American Academy of child and Adolescent Psychiatry, 39, 28-38.

Simons, M., Schneider, S. y Herpertz-Dahlmann, B. (2006). Metacognitive Therapy versus Exposure and Response Prevention for Pediatric Obsessive-Compulsive Disorder. Psychotherapy and Psychosomatics, 75, 257264
Storch, E.A., Geffken, G.R., Merlo, L.J., Mann, G., Duke, D., Munson, M., Adkins, J., Grabill, K.M., Murphy, T.K. y Goodman, W.K. (2007). Family-based cognitive-behavioral therapy for pediatric obsessivecompulsive disorder: Comparison of intensive and weekly approaches. Journal of the American Academy of Child and Adolescent Psychiatry, 46, 469478.

Storch, E.A., Merlo, L.J., Larson, M.J., Bloss, C.S., Geffken, G. R., Jacob, M.L., Murphy, T.K., y Goodman, W.K. (2008). Symptom dimensions and cognitive-behavioural therapy outcome for pediatric obsessivecompulsive disorder. Acta Psychiatrica Scandinava, 117, 67-75.

Storch, E.A., Merlo, L.J., Larson, M.J., Marien, W.E., Geffken, G.R., Jacob, M.L., Goodman, W.K. y Murphy, T. K. (2008). Clinical features associated with treatment-resistant pediatric obsessive-compulsive disorder. Comprehensive Psychiatry, 49, 35-42.

Storch, E.A, Murphy, T.K., Geffken, G.R., Mann, G., Adkins, J., Merlo, L.J., Duke, D., Munson, M., Swaine, Z. y Goodman, W.K. (2006). Cognitive-Behavioral Therapy for PANDAS-Related Obsessive-Compulsive Disorder: Findings From a Preliminary Waitlist Controlled Open Trial. Journal of the American Academy of Child and Adolescent Psychiatry, 45, 11711178.

Thienemann, M., Martin, J., Cregger, B., Thompson, H., y Dyer-Friedman, J. (2001). Manual-driven group cognitive-behavioural therapy for adolescents with obsessive-compulsive disorder: A pilot study. Journal of the American Academy of Child and Adolescent Psychiatry, 40, 1254-1260.

Toro, J. (2006). Trastornos obsesivos en la infancia y la adolescencia. En J. Vallejo y G. E. Berrios (eds.), Estados obsesivos (pp. 433-459). Barcelona: Masson.

Turner, C., Heyman, I., Futh, A. y Lovell, K. (2009). A pilot study of telephone cognitive-behavioural therapy for obsessive-compulsive disorder in young people. Behavioural and Cognitive Psychotherapy, 37, 469-474.

Valderhaug, R., Larssona, B., Götestamb, K.G. y Piacentini, J. (2007). An open clinical trial of cognitive-behaviour therapy in children and adolescents with obsessive-compulsive disorder administered in regular outpatient clinics. Behaviour Research and Therapy, 45, 577-5897.

Wagner, A.P. (2003). Cognitive-behavioral therapy for children and adolescentswith obsessive compulsive disorder. Brief Treatment and Crisis Intervention, 3, 291-306.

Waters, T. L., Barrett, P. y March, J. S. (2001). Cognitive-behavioral family treatment of childhood obsessive-compulsive disorder: Preliminary findings. American Journal of Psychotherapy, 55, 372-387.

Watson, H.J. y Rees, C.S. (2008). Meta-analysis of randomized, controlled treatment trials for pediatric obsessive-compulsive disorder. Journal of Child Psychology and Psychiatry and Allied Disciplines, 49, 489-498.

Wells, A. (1997). Cognitive therapy of anxiety disorders: A practice manual and conceptual guide. Chichester, Reino Unido: John Wiley and Sons.

Whiteside, S.P y Abramowitz, J.S. (2006). Relapse Following Successful Intensive Treatment of Pediatric Obsessive-Compulsive Disorder: A Case Study. Clinical Case Studies, 5, 522-540.

Whiteside, S.P, Brown, A.M y Abramowitz, J.S. (2008). Five-day intensive treatment for adolescent OCD: A case series. Journal of Anxiety Disorders, 22, 495-504.

Williams, T.I, Salkovskis, P., Forrester, E. y Allsopp, M. (2002). Changes in symptoms of OCD and appraisal of responsibility during cognitivebehavioural treatment: A pilot study. Behavioural and Cognitive Psychotherapy, 30, 69-78.

Williams, T.I., Salkovskis, P.M, Forrester, L., Turner, S., White, H. y Allsop, M.A. (2010). A randomised controlled trial of cognitive behavioural tratment for obsessive compulsive disorder in children and adolescents. European of Children and Adolescents Psychiatry, 19, 449-456.

(Articulo recibido: 16-03-2011, revisión: 25-10-2011, aceptado: 26-10-2011) 